\title{
Off-axis beam dynamics in rf-gun-based electron photoinjectors
}

\author{
R. Huang, ${ }^{1,2}$ C. Mitchell,,${ }^{1, *}$ C. Papadopoulos, ${ }^{1}$ H. Qian, ${ }^{1}$ M. Venturini, ${ }^{1}$ \\ J. Qiang, ${ }^{1}$ D. Filippetto, ${ }^{1}$ J. Staples,${ }^{1}$ Q. Jia, ${ }^{2}$ and F. Sannibale ${ }^{1}$ \\ ${ }^{1}$ Lawrence Berkeley National Laboratory, One Cyclotron Road, Berkeley, California 94720, USA \\ ${ }^{2}$ NSRL, University of Science and Technology of China, Hefei, Anhui 230029, China
}

(Received 1 April 2016; published 22 November 2016)

\begin{abstract}
The need to operate an rf-gun-based electron photoinjector with a beam emitted away from the cathode center can occur under various circumstances. First, in some cases the cathode can be affected by ion backbombardment that progressively reduces the quantum efficiency $(\mathrm{QE})$ in its center, making off-axis operation mandatory; second, in some cases the drive laser intensity can be sufficiently high to generate QE depletion in the cathode area illuminated by the laser, forcing off-axis operation; last, in cathodes with nonuniform QE distribution it could be convenient to operate off axis to exploit a better QE. However, operation in this mode may lead to growth of the projected transverse beam emittances due to correlations between the transverse and longitudinal degrees of freedom that are introduced within the gun and downstream rf cavities. A strategy is described to mitigate this emittance growth by allowing the beam to propagate along a carefully tuned off-axis trajectory in downstream rf cavities to remove the timedependent rf kicks introduced in the gun. Along this trajectory, short range wakefields do not degrade the emittance, and long range wakefields degrade the emittance for very high repetition rate only.
\end{abstract}

DOI: 10.1103/PhysRevAccelBeams.19.113401

\section{INTRODUCTION}

Rf photoguns have made a tremendous impact on the range of applications of electron accelerators, improving the source beam brightness by orders of magnitude with respect to previous generations of electron sources. The combination of high peak fields at the cathode with sub$\mathrm{mm}$ spot size and sub-ps laser pulses has enabled the production of short high current and low emittance beams that can be used to drive 4th generation light sources [1], generate copious amounts of $\mathrm{THz}$ radiation [2], probe structural dynamics at the ultrafast time scale [3], or serve as injectors for laser-based compact accelerators [4].

Typically in rf photoguns, a laser stimulates photoemission by illuminating the cathode at its center. In this situation, the emitted beam and the applied electromagnetic fields possess cylindrical symmetry about the direction of motion, producing high-quality performance in terms of the electron beam emittance (and hence in transverse brightness). Nevertheless, special circumstances can force one to operate the laser away from the cathode center. For example, in some cases, especially in high duty cycle or continuous wave (CW) rf accelerators, residual gas molecules ionized by the photo-emitted electrons can be accelerated back to the central area of the cathode,

*chadmitchell@lbl.gov

Published by the American Physical Society under the terms of the Creative Commons Attribution 3.0 License. Further distribution of this work must maintain attribution to the author(s) and the published article's title, journal citation, and DOI. generating local sputtering that can progressively reduce the quantum efficiency $(\mathrm{QE})$ in the cathode center $[5,6]$. In other cases, the drive laser intensity required to generate high charge bunches at high repetition rates can be sufficiently large to generate progressive $\mathrm{QE}$ depletion in the cathode area illuminated by the laser. In this case, offaxis illumination can be used to prolong the QE lifetime of the cathode. Finally, in the presence of cathodes with nonuniform QE distribution, it could be convenient to operate in off-axis areas with higher QE. The Cornell DC gun makes use of a photocathode [7] with an active area off-center to avoid ion damage, and measurements show impressive QE preservation [8]. The challenge in using offaxis emission at the cathode is the potential growth in beam projected emittance that such configurations can induce. This is particularly true in the case of rf guns, where timedependent focusing effects due to the gun fields can generate distribution asymmetries and longitudinaltransverse correlations that will ultimately increase the beam projected emittance. In this paper, we investigate this effect analytically and through simulations, and we define a correction procedure that can largely compensate for the induced projected emittance growth. We also include an analysis of the role that transverse wakefields play in this scenario. For all simulations we use an rf photoinjector based on the very-high-frequency $(186 \mathrm{MHz})$ gun developed at Lawrence Berkeley National Laboratory in the framework of the Advanced Photoinjector EXperiment (APEX) [9]. However, the strategies we describe here can in principle be applied to any electron injector using a rf photogun. 


\section{TIME-DEPENDENT FOCUSING IN RF CAVITIES}

An electron bunch propagating off-axis through a rf cavity experiences a time-dependent transverse kick due to the time-varying cavity fields. As a result, different longitudinal slices within the bunch will be transported to different locations in the transverse phase plane at the cavity exit. This effect generates a correlation between transverse and longitudinal degrees of freedom within the bunch, resulting in an increase of the projected beam emittances. In this section, we discuss a simple model for this effect and describe how it can be corrected.

\section{A. Correlations introduced during off-axis operation}

Consider a single mode of frequency $f$ in a rf cavity with a time-dependent longitudinal on-axis electric field described by:

$$
E_{z}(z, t)=e(z) \cos \left(\omega t+\phi_{0}\right)=\mathcal{R} e\left[e(z) e^{i\left(\omega t+\phi_{0}\right)}\right],
$$

where $\omega=2 \pi f, e(z)$ describes the spatial dependence of the field on-axis, and $\phi_{0}$ denotes the cavity phase at $t=0$. In the discussion that follows, we will use the complex notation appearing in the rightmost expression of (1), where the notation indicating the real part will be omitted.

One can show directly from Maxwell's equations that in the special case when the fields are cylindrically symmetric $[10,11]$ :

$$
\begin{gathered}
E_{r}=-\frac{r}{2} \frac{\partial E_{z}}{\partial z}=-\frac{r}{2} e^{\prime}(z) e^{i\left(\omega t+\phi_{0}\right)}+O\left(r^{3}\right), \\
c B_{\phi}=\frac{r}{2 c} \frac{\partial E_{z}}{\partial t}=\frac{i r \omega}{2 c} e(z) e^{i\left(\omega t+\phi_{0}\right)}+O\left(r^{3}\right),
\end{gathered}
$$

where the symbol ' indicates differentiation with respect to $z$. The transverse Lorentz force acting on a particle in the cavity is therefore given by:

$$
\begin{aligned}
F_{r} & =q\left(E_{r}-\beta c B_{\phi}\right) \\
& =-\frac{q r}{2}\left\{e^{\prime}(z)+i \beta \frac{\omega}{c} e(z)\right\} e^{i\left(\omega t+\phi_{0}\right)}+O\left(r^{3}\right),
\end{aligned}
$$

where $\beta$ is the ratio of the particle speed to the speed of light $c$. Detailed information about representing rf electric and magnetic fields to higher order, including the more general case without cylindrical symmetry, can be found in [12].

In the simplest approximation, suppose that a particle with zero initial transverse momentum travels through the cavity along an unperturbed straight-line trajectory at a fixed distance $r$ from (and parallel to) the symmetry axis. Such a particle will acquire nonzero transverse momentum due to the impulsive kick caused by rf defocusing in the cavity. Here, we will assume that all momenta are normalized by the factor $m c$, and are therefore dimensionless. Expanding the final transverse momentum of the particle to second order in the distance $r$ gives:

$$
p_{r}=\frac{1}{m c} \int_{t_{i}}^{t_{f}} F_{r}(t) d t=r\left(\frac{\partial p_{r}}{\partial r}\right)+O\left(r^{3}\right) .
$$

It follows from (3) that the second-order term in $r$ vanishes. Without additional approximation, evaluation of the coefficient appearing in (4) requires numerical integration, and the result depends on the particle energy and the phase at which the particle crosses the cavity. For approximate treatments, see $[10,13]$. Consider now a cold (monoenergetic) bunch of finite length, so that particles at different longitudinal locations within the bunch will cross the cavity with slightly different phases. Expanding with respect to the longitudinal coordinate $z$ relative to the bunch centroid location then gives:

$$
p_{r}=r\left(\frac{\partial p_{r}}{\partial r}+\frac{\partial^{2} p_{r}}{\partial r \partial z} z+O\left(z^{2}\right)\right)+O\left(r^{3}\right),
$$

where the coefficients in (5) are now determined by the reference phase of the bunch centroid. Notice that (5) takes the form of a second-degree Taylor map based on the variables $r$ and $z$.

Consider a beam with no initial transverse momentum spread and no initial transverse-longitudinal correlations, $\langle\Delta x \Delta z\rangle=\langle\Delta y \Delta z\rangle=0$. We allow the beam to propagate through the rf cavity with an initial centroid offset $\left(x_{0}, y_{0}\right)$. One can verify directly from (5) that the beam acquires a transverse momentum spread of the form:

$$
\begin{aligned}
& \left\langle\Delta p_{x}^{2}\right\rangle=\left(\frac{\partial p_{r}}{\partial r}\right)^{2} \sigma_{x}^{2}+\left(\frac{\partial^{2} p_{r}}{\partial r \partial z}\right)^{2}\left(x_{0}^{2}+\sigma_{x}^{2}\right) \sigma_{z}^{2}, \\
& \left\langle\Delta p_{y}^{2}\right\rangle=\left(\frac{\partial p_{r}}{\partial r}\right)^{2} \sigma_{y}^{2}+\left(\frac{\partial^{2} p_{r}}{\partial r \partial z}\right)^{2}\left(y_{0}^{2}+\sigma_{y}^{2}\right) \sigma_{z}^{2},
\end{aligned}
$$

and furthermore, this acquired momentum is correlated with position:

$$
\begin{gathered}
\frac{\left\langle\Delta p_{w} \Delta z\right\rangle}{\sigma_{z}^{2}}=w_{0}\left(\frac{\partial^{2} p_{r}}{\partial r \partial z}\right) \quad \text { for } w=x, y, \\
\frac{\left\langle\Delta p_{x} \Delta x\right\rangle}{\sigma_{x}^{2}}=\frac{\left\langle\Delta p_{y} \Delta y\right\rangle}{\sigma_{y}^{2}}=\left(\frac{\partial p_{r}}{\partial r}\right) .
\end{gathered}
$$

This gives an expression for the final rf-induced horizontal beam emittance of the form [14]:

$$
\varepsilon_{x, n}=\left|\frac{\partial^{2} p_{r}}{\partial r \partial z}\right| \sigma_{z} \sigma_{x} \sqrt{\sigma_{x}^{2}+x_{0}^{2}} .
$$

An equivalent expression is found for the vertical plane. In the following subsection, we will evaluate the coefficient 
appearing in (9) for a realistic system, and we will show how this emittance growth can be corrected.

\section{B. Correlation removal using a downstream cavity}

Consider a simple system consisting of an rf gun followed by a single downstream cavity. As an illustration, we will use the rf gun together with the buncher cavity shown in the photoinjector beam line of Fig. 1. For a beam emitted off-axis at the cathode, we can remove the timedependent correlation introduced by the gun by steering the beam offset in the buncher so that the total correlation introduced by the two cavities is zero:

$$
\boldsymbol{r}_{\boldsymbol{G}}\left(\frac{\partial^{2} p_{r}}{\partial r \partial z}\right)_{G}+\boldsymbol{r}_{\boldsymbol{B}}\left(\frac{\partial^{2} p_{r}}{\partial r \partial z}\right)_{B}=0
$$

Here $\boldsymbol{r}_{\boldsymbol{G}}$ and $\boldsymbol{r}_{\boldsymbol{B}}$ denote the transverse coordinates of the beam centroid at the entrance to the rf gun and the buncher, respectively. Here, we assume that ideal steering allows one to control exactly the centroid offset at the buncher entrance. Furthermore, we assume that the bunch length is approximately fixed, and that there is no active solenoid between the gun and buncher cavity. Larmor rotation of $\boldsymbol{r}_{\boldsymbol{G}}$ is required if a solenoid field is present.

From (8a) we see that the $z-p_{x}$ correlation introduced within each cavity is proportional to the centroid offset $x_{0}$ at the cavity entrance. For each cavity, one can evaluate the coefficient $\left(\partial^{2} p_{r} / \partial r \partial z\right)$ by tracking beams through the cavity for several different values of initial centroid offset. For the system consisting of the gun and the buncher cavity of Fig. 1, we calculated the correlation introduced in each cavity by using two separate beams, as shown in Fig. 2. The beam entering each cavity has no initial $z-p_{x}$ correlation, but is given an initial horizontal offset $x_{0}$. We used a beam distribution entering each cavity that is identical to the onaxis beam distribution obtained at the same location in the beam line of Fig. 1. The beam in the gun is accelerated to an energy of $800 \mathrm{keV}$, while the buncher cavity is operated at a phase corresponding to zero crossing. It is clear that the $z-p_{x}$ correlation acquired in each cavity scales linearly with the centroid offset at the cavity entrance. The coefficients $\left(\partial^{2} p_{r} / \partial r \partial z\right)$ are the slopes of the lines in Fig. 2, which depend on the cavity gradient, phase, and initial beam energy [13]. In our case, the coefficients are $-23.8 \mathrm{mc} /$ meter $^{2}$ and $7.90 \mathrm{mc} /$ meter $^{2}$ (normalized by $m c$ ) for the gun and the buncher cavity, respectively. This indicates that the development of a $z-p_{x}$ correlation in the beam is more sensitive to a transverse offset in the gun than to a transverse offset in the buncher cavity.

Using (10), the relationship between the initial beam offset at the cathode and the offset in the downstream buncher cavity that is required to remove the $z-p_{x}$ correlation is given by:

$$
\frac{r_{B}}{r_{G}}=-\frac{\left(\partial^{2} p_{r} / \partial r \partial z\right)_{G}}{\left(\partial^{2} p_{r} / \partial r \partial z\right)_{B}}
$$

For typical APEX gun parameters, which will be specified in the following section, this requires that the beam enter the buncher with a transverse offset about 3 times the offset at the cathode. To verify this simple two-cavity model, we simulated a beam in the APEX gun emitted with $2 \mathrm{~mm}$ horizontal offset from the cathode center, tracking the beam to the exit of the buncher. For simplicity, the solenoid between the gun and buncher is turned off in this simulation. The $z-p_{x}$ correlation in the beam at the buncher exit is observed to decrease to zero when the beam is steered to a $6.2 \mathrm{~mm}$ offset entering the buncher, consistent with this simple analytical model. We also expect from (9) that by removal of these time-dependent correlations, the final emittance of the beam emitted off-axis can be sharply reduced. In this case, the simulated horizontal projected emittance at the exit of the buncher was reduced from $1.268 \mu \mathrm{m}$ to $0.423 \mu \mathrm{m}$ by this correction procedure, while the vertical emittance remains similar to that of a beam emitted on-axis $(0.227 \mu \mathrm{m})$. Note that the steering correction needs to be performed at low energy to avoid the need for a large transverse offset.

The analytical model described in this section only includes the effect of the linear $z-p_{r}$ correlation along the beam that occurs due to time-dependent rf focusing when the beam is emitted off-axis. In general, this is the dominant contribution to the beam emittance. However, there are additional correlations introduced to the beam that result from feed-down due to other second-order terms in the transfer map for the rf gun, and accurate simulation is

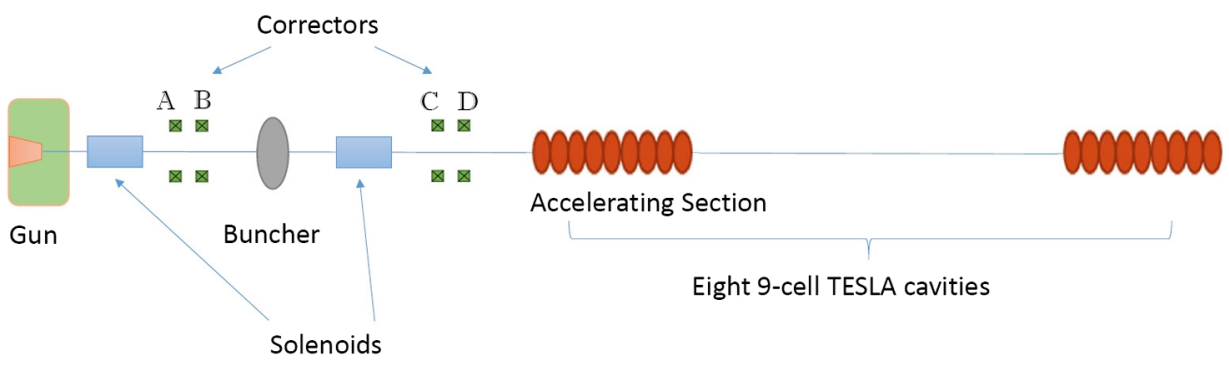

FIG. 1. Schematic of the photoinjector based on the APEX VHF-Gun used for these studies. The injector exit is located $15 \mathrm{~m}$ downstream from the cathode. 

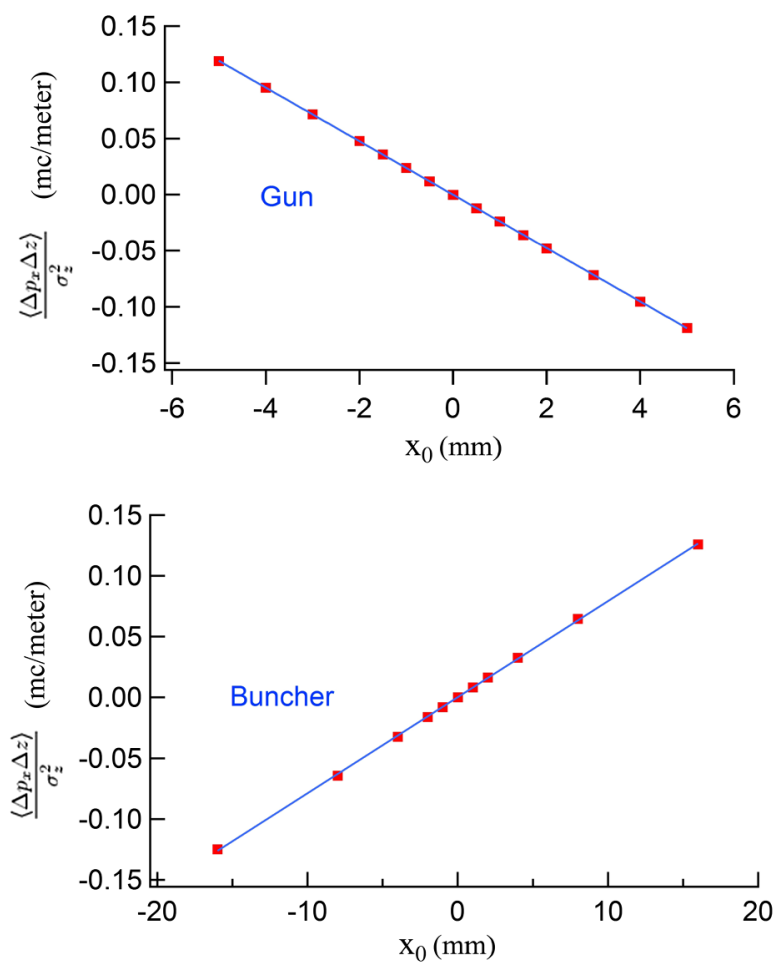

FIG. 2. $z-p_{x}$ correlation introduced by a rf cavity for different initial offsets. An initially uncorrelated beam enters each cavity with an initial horizontal offset $x_{0}$. The final $z-p_{x}$ correlation is calculated at the exit of each cavity, using ASTRA simulation. The upper figure shows the correlation introduced by the rf gun and the lower shows the correlation introduced by the rf buncher cavity. The lines denote linear fits to the simulation data, with the slopes defining the correlation coefficients $\left(\partial^{2} p_{r} / \partial r \partial z\right)$.

required to evaluate the resulting contributions to the beam emittance. A detailed investigation of emittance growth will be discussed in the next two sections.

\section{BEAM SIMULATIONS USING AN RF PHOTOINJECTOR LAYOUT}

\section{A. Beam propagation in an APEX-based photoinjector}

For investigating the effects of the theory explained in the previous section, we use in our simulations a photoinjector based on APEX, the injector R\&D facility developed at LBNL for testing the performance of the high brightness, high repetition rate VHF-Gun. In the layout used for the simulations, the room-temperature accelerating sections of APEX are replaced by 8 Tesla-type superconducting cavities [15] to better represent the situation of an actual high-repetition rate photoinjector. Figure 1 shows a schematic view of the photoinjector showing the elements relevant to the simulations. The same layout was previously used for simulations for the LCLS-II injector [16]. The parameters of the beamline components used for the studies are included in Table I.

TABLE I. The parameters of the beam line components relevant to these studies.

\begin{tabular}{lc}
\hline \hline Gun rf frequency & $186 \mathrm{MHz}$ \\
Gun rf gap & $4 \mathrm{~cm}$ \\
Beam energy at gun exit & $800 \mathrm{keV}$ \\
Gun field at nominal energy & $20 \mathrm{MV} / \mathrm{m}$ \\
Buncher frequency & $1.3 \mathrm{GHz}$ \\
Maximum field in buncher & $3.67 \mathrm{MV} / \mathrm{m}$ \\
Accelerating cavity frequency & $1.3 \mathrm{GHz}$ \\
Maximum field in accelerating sections & $29.5 \mathrm{MV} / \mathrm{m}$ \\
Energy gain at nominal power & $95 \mathrm{MeV}$ \\
Solenoid 1 maximum field on-axis & $0.0422 \mathrm{~T}$ \\
Solenoid 2 maximum field on-axis & $0.0292 \mathrm{~T}$ \\
Gun beam exit pipe diameter & $2.8 \mathrm{~cm}$ \\
Buncher iris diameter & $5.0 \mathrm{~cm}$ \\
Tesla cavity iris diameter & $7.0 \mathrm{~cm}$ \\
\hline \hline
\end{tabular}

The beam used in simulation consists of $100 \mathrm{pC}$ charge per bunch, with an initial transverse uniform distribution and longitudinal plateau distribution. Major beam parameters for the simulation are listed in Table II. A multi-objective genetic algorithm (MOGA) combining the algorithm NSGA-II [17] with the beam dynamics tool ASTRA [18] was used to obtain the settings for the gun, the solenoids, and the rf cavities that are needed to minimize the final transverse beam emittance and bunch length in the case of an on-axis beam. The complete optimization procedure is reported elsewhere [16]. The injector setup is based on one such optimized solution, and the settings are identical for both the on-axis and off-axis beam simulations reported in this section. Once these settings are obtained, simulations are performed using the code IMPACT-T [19], which is chosen due to its fast and accurate treatment of 3D space charge.

To evaluate the beam dynamics in an extreme case, we use a beam emitted $2 \mathrm{~mm}$ horizontally off-axis at the cathode, a value that is ten times the rms laser spot size, as shown in Fig. 3. After being emitted off-axis, the beam will propagate along the beamline with the transverse centroid evolution shown in the top plot of Fig. 4. Compared with the on-axis beam, the beam emitted off-axis remains offaxis downstream, and it will go off-axis as far as $7.3 \mathrm{~mm}$ in the first solenoid and $4.1 \mathrm{~mm}$ at the injector exit. It is notable that the misalignment is transferred from horizontal

TABLE II. Beam parameters used in simulation.

Bunch charge

Transverse distribution

Laser spot size (rms)

Thermal emittance (rms)

Longitudinal distribution

Bunch length (FWHM)

Rise and fall time

Kinetic energy at injector exit
$100 \mathrm{pC}$

Radial uniform

$192 \mu \mathrm{m}$

$1.0 \mu \mathrm{m} / \mathrm{mm}$

Plateau

33 ps

2 ps $95 \mathrm{MeV}$ 

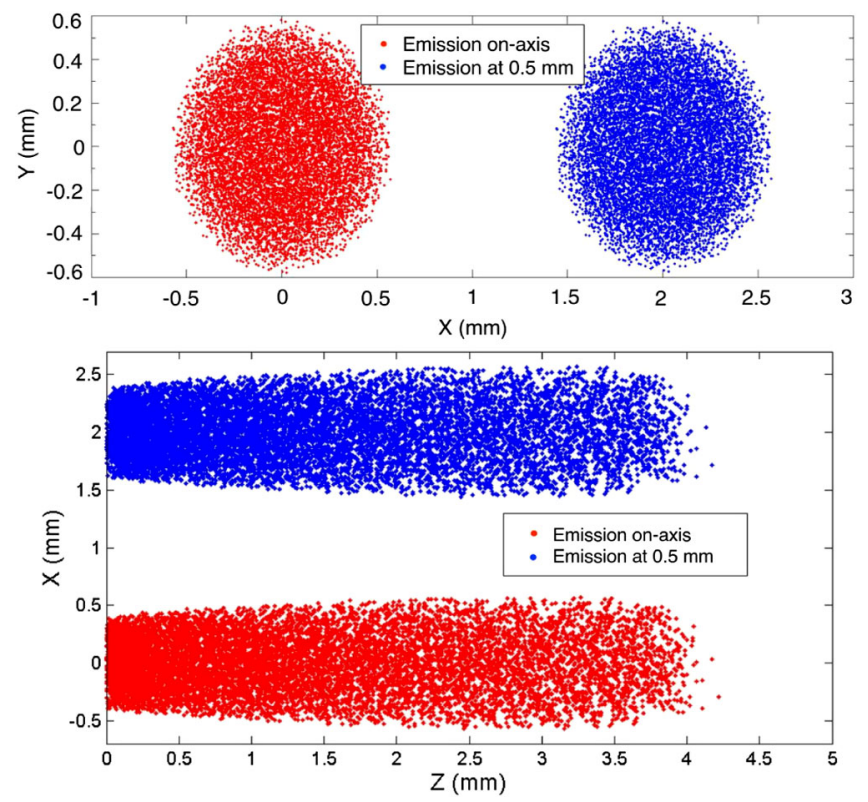

FIG. 3. Particle distribution at the cathode. Red dots are particles emitted on-axis near the center of the cathode. Blue dots are particles emitted off-axis with $2.0 \mathrm{~mm}$ horizontal misalignment. (Upper) X-Y projection. (Lower) Z-X projection.

to vertical due to the two solenoids that rotate the beam by almost 90 degrees. The emittance evolution of both the on-axis and the off-axis beams is shown in the bottom plot of Fig. 4.

The final emittance of the beam emitted $2 \mathrm{~mm}$ off-axis is increased by $20 \%$ and $168 \%$ in the horizontal and vertical, respectively, relative to the emittance of the on-axis beam of $0.342 \mu \mathrm{m}$. Figure 5 illustrates that this difference begins at low energy, in the gun and in the first solenoid. The particle distribution at the exit of the injector is shown in Fig. 6. We see that the beam's cylindrical symmetry is destroyed by transverse rf kicks that occur because the beam is not passing through the center of the gun and the rf cavities. An evident correlation is visible between the vertical plane and longitudinal position, resulting in an increased vertical projected emittance. This illustrates that driving the laser far off-center in the rf gun will lead to severe beam emittance dilution. The emittance increase is smaller for smaller initial beam offset. For example, for a beam with $0.5 \mathrm{~mm}$ initial horizontal offset (2.5 times the rms laser spot size), the final emittance is only $0.6 \%$ larger in the horizontal and $11.4 \%$ larger in the vertical, relative to the on-axis beam.

\section{B. Projected emittance decomposition}

To better understand the projected (rms) beam emittance growth in terms of the various longitudinal beam slices, the projected emittance can be decomposed into four contributions corresponding to distinct dynamical processes [20]. The horizontal and vertical planes are

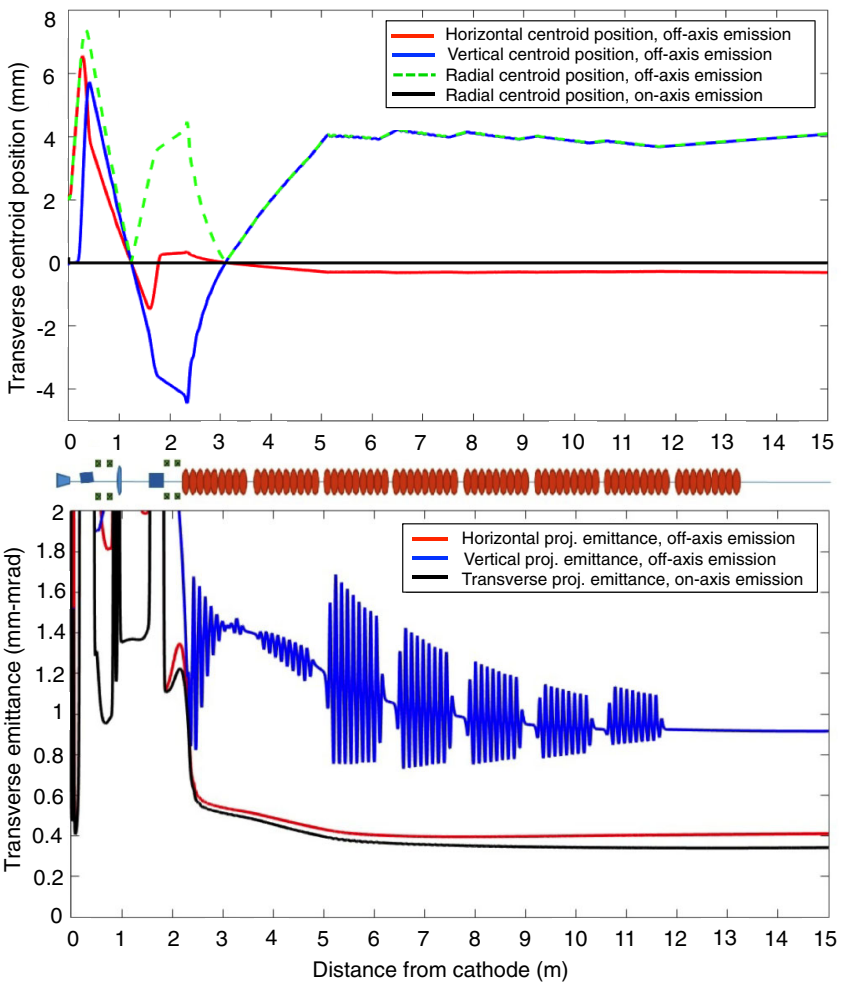

FIG. 4. Evolution of the transverse centroid (upper) and transverse emittance (lower) along the length of the injector system of Fig. 1. The red and blue lines are, respectively, the horizontal and vertical results for a beam emitted $2.0 \mathrm{~mm}$ horizontally off-axis. Results for the on-axis beam are shown in black.

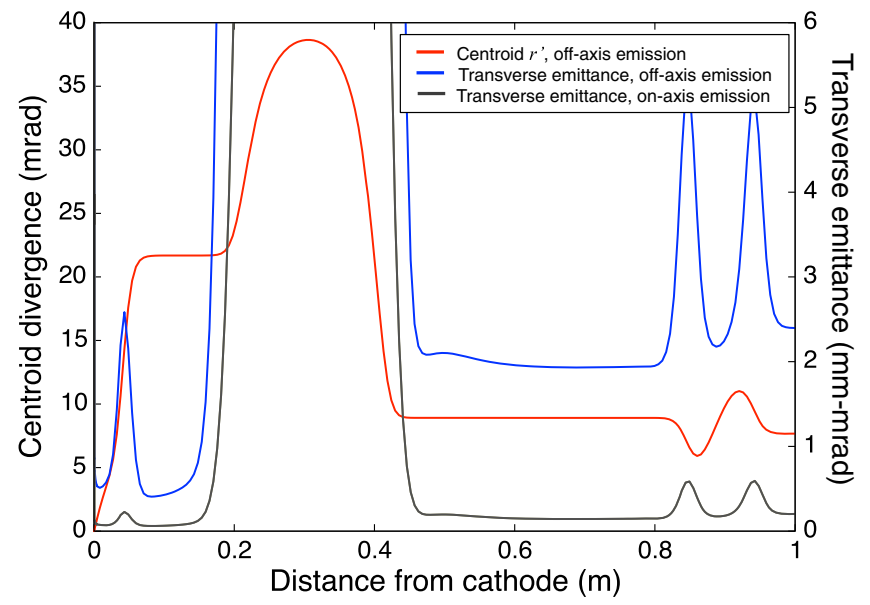

FIG. 5. A close-up view of the behavior in Fig. 4 from the cathode to the exit of the rf buncher, illustrating the effects of $\mathrm{rf}$ defocusing in the gun. (Red) Evolution of the centroid divergence angle $r^{\prime}=p_{r} / p_{z}$ for a beam emitted $2 \mathrm{~mm}$ horizontally off-axis at the cathode. (Blue) Geometric mean of the horizontal and vertical normalized transverse emittances for a beam emitted $2 \mathrm{~mm}$ horizontally off-axis at the cathode. (Black) The corresponding transverse emittance for a beam emitted on-axis at the cathode. 

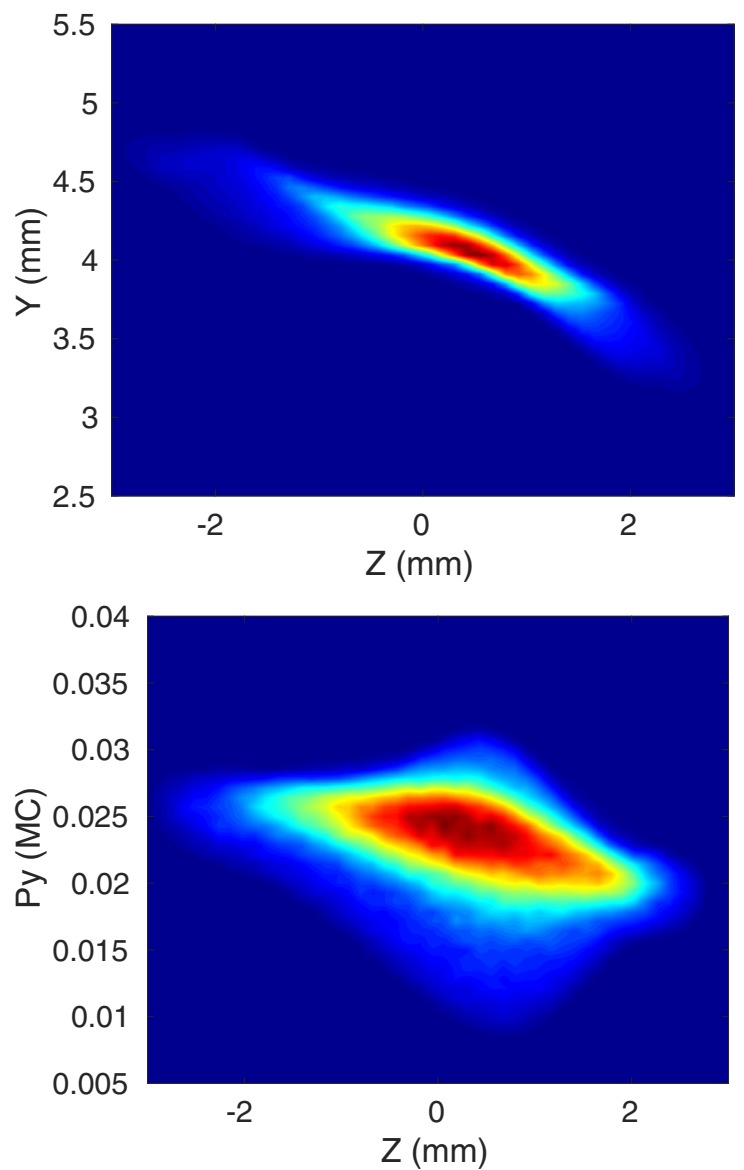

FIG. 6. Particle distribution of a beam emitted $2.0 \mathrm{~mm}$ off-axis at the cathode, after tracking to the exit of the injector shown in Fig. 1. Transverse-longitudinal correlations are visible in the final beam phase space. Here color denotes particle density, with red-black denoting regions of highest particle density and blue denoting regions of lowest particle density. (Upper) Z-Y projection. (Lower) Z-Py projection.

coupled because of Larmor rotation, so that the primary effect of horizontal off-axis emission is to increase the vertical projected emittance. The decomposition of the vertical projected emittance is given by an expression of the form:

$$
\varepsilon^{2}=\varepsilon_{\perp}^{2}+\varepsilon_{R}^{2}+\varepsilon_{\mathrm{int}}^{2}+\varepsilon_{\|}^{2} .
$$

A mathematical description of the four terms appearing in (12) is provided in [20]. Here $\varepsilon_{\perp}$ is the average slice emittance, and $\varepsilon_{R}$ is the emittance contribution due to variation of the slice Twiss functions along the length of the bunch ("mismatch" of the slice phase space ellipses). The third contribution $\varepsilon_{\text {int }}$ is due to misalignment of the slice centroids in the transverse phase space. Finally, the contribution $\varepsilon_{\|}$occurs when there is a strong nonlinear transverse-longitudinal correlation, usually due to the effects of a longitudinal wakefield in a region with nonvanishing dispersion [21]. The contribution $\varepsilon_{\|}$
TABLE III. Decomposition of the vertical projected emittance for a beam emitted on-axis and for a beam emitted $2 \mathrm{~mm}$ off-axis, evaluated at the exit of the photoinjector beam line shown in Fig. 1. The percentage contribution of each term to the sum in quadrature (12) is also given. Space charge is turned off.

\begin{tabular}{lcc}
\hline \hline Emittance contribution $(\mu \mathrm{m})$ & $\begin{array}{c}\text { On-axis } \\
\text { beam }\end{array}$ & $\begin{array}{c}\text { Off-axis emitted } \\
\text { beam }\end{array}$ \\
\hline Projected emittance $\varepsilon$ & 0.1958 & 0.4854 \\
Mean slice $\varepsilon_{\perp}$ & $\mathbf{0 . 1 8 9 5}$ & $\mathbf{0 . 2 0 2 0}$ \\
& $(95.4 \%)$ & $(17.4 \%)$ \\
Mismatch $\varepsilon_{R}$ & $\mathbf{0 . 0 3 8 1}$ & $\mathbf{0 . 0 4 4 5}$ \\
Linear correlation $\varepsilon_{\text {int }}$ & $(3.9 \%)$ & $(0.8 \%)$ \\
Nonlinear correlation $\varepsilon_{\|}$ & $\mathbf{0 . 0 1 3 0}$ & $\mathbf{0 . 4 3 7 7}$ \\
& $(0.5 \%)$ & $(81.7 \%)$ \\
& $\mathbf{0 . 0 0 0 1}$ & $\mathbf{0 . 0 1 3 7}$ \\
& $(0.0 \%)$ & $(0.1 \%)$ \\
\hline \hline
\end{tabular}

vanishes when there is a purely linear correlation between the transverse and longitudinal planes.

We simplify the problem by simulating the beam through the beam line of Fig. 1 without including space charge. For the beam emitted on-axis, the final vertical projected emittance is $0.1958 \mu \mathrm{m}$, with contributions given by $\varepsilon_{\perp}=0.1895 \mu \mathrm{m}, \varepsilon_{R}=0.0381 \mu \mathrm{m}$, $\varepsilon_{\text {int }}=0.0130 \mu \mathrm{m}$, and $\varepsilon_{\|}=0.0001 \mu \mathrm{m}$. The average slice emittance $\varepsilon_{\perp}$ is the dominant contribution to the projected emittance. (For the best-case scenario, all slices of the beam have identical ellipses in the transverse phase space. In this case, the final three emittance contributions vanish and $\varepsilon_{\perp}$ gives the projected emittance of the entire beam.) We also performed a decomposition of the final vertical emittance for a beam emitted $2.0 \mathrm{~mm}$ horizontally off-axis, and the result is given in Table III for comparison with the on-axis case. Note that the projected emittance values for both the off-axis and on-axis cases are smaller than those given in the previous section, where space charge was included. Comparing the final two columns of Table III, we see that the large difference in projected emittance is due almost entirely to the contribution $\varepsilon_{\text {int }}$. This indicates that the increase in projected emittance is due to the misalignment of slice centroids caused by time-dependent rf kicks in the rf gun and accelerating cavities. Furthermore, one can verify that the correlation contribution $\varepsilon_{\text {int }}$ is introduced in the rf gun, since this term is dominant when the beam arrives at the gun exit. Using this formalism, we can distinguish between the various dynamical sources of emittance growth.

Although initial beam spot displacement causes remarkable emittance growth and misalignment in the downstream components, it is possible to correct beam misalignment by steering the beam in a magnetic field. A practical correction procedure for emittance compensation and transverselongitudinal correlation reduction will be proposed in the next section. 


\section{BEAM CORRECTION PROCEDURES}

As discussed in the previous section, operating the beam with a transverse offset is detrimental to the beam emittance. In this section, we investigate possible correction procedures to reduce this source of emittance growth.

\section{A. Aligning the beam centroid to the beam line axis}

As a first attempt, one can try to reduce emittance growth for a beam emitted off-axis by using dipole correctors to realign the beam onto the beam line axis immediately after the gun exit. In this section, we will evaluate whether this method is effective.

In the APEX beam line, the first solenoid is equipped with remotely controlled motors that allow for adjustment of both its position and orientation angle. Because of this, it is possible to align the solenoid to the orbit of the off-axis beam coming from the gun, such that the beam passes through the solenoid along its axis. This allows the beam to avoid sampling the nonlinear fields that occur far from the solenoid axis. Additionally, the pair of horizontal and vertical dipole corrector magnets located between the first solenoid and the buncher cavity can be used to align the beam centroid in both position and angle before entering the buncher and downstream beamline components.

The code ASTRA was used to simulate a $100 \mathrm{pC}$ beam emitted $2 \mathrm{~mm}$ off-axis at the cathode, after correction using the alignment procedure just described. The simulation results show that, in spite of the beam realignment along the entire beam line using the correctors, the final emittance of the beam at the injector exit remains significantly larger than the final emittance of a beam emitted on-axis. Thus, using dipole correctors to realign the beam onto the beam line axis fails to eliminate the projected emittance growth, due primarily to the effects of time-dependent rf focusing in the gun, which cannot be compensated by the static magnetic fields of the corrector dipoles.

\section{B. Optimizing the beam orbit for minimum emittance}

In Sec. II, it was shown that the rf-induced beam emittance increase associated with the beam offset in the gun can be compensated by properly adjusting the beam orbit inside a downstream rf cavity. In this section, we exploit this effect to minimize the emittance at the exit of the injector system shown in Fig. 1.

In order to obtain a beam with minimum emittance at the injector exit, the magnetic field strengths in the steering coils (referred to as correctors) upstream of the buncher cavity were varied to find a compensating beam orbit through the buncher and the accelerating sections. A MOGA approach was used to minimize the final beam emittance, with the correctors' strengths used as control parameters (knobs). All the other injector parameters were held fixed, including the rf gradient and phase of the gun, of

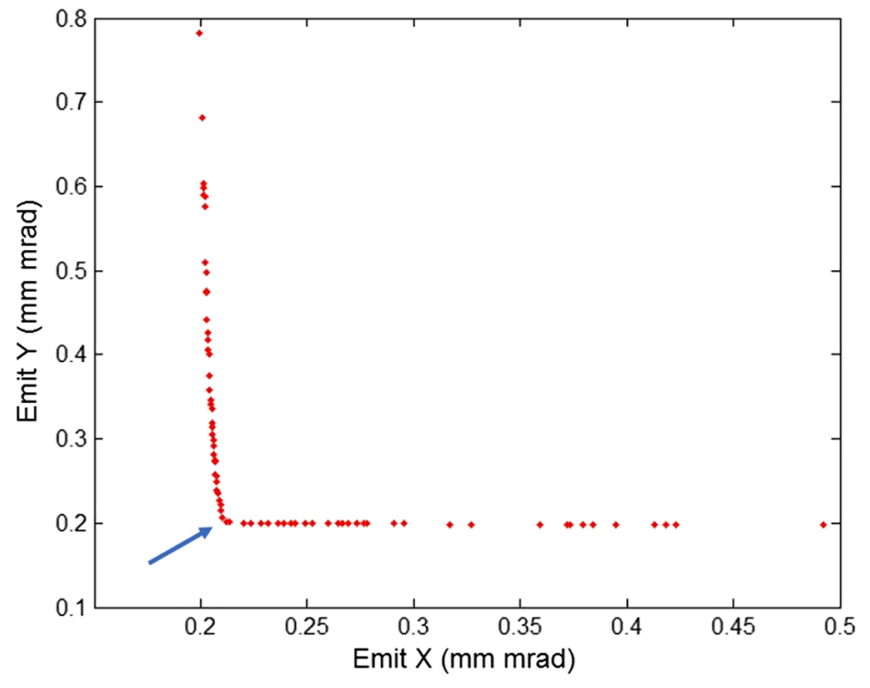

FIG. 7. A Pareto-optimal front [22] showing the set of solutions that minimize the horizontal and vertical normalized emittances at the injector exit obtained by varying the strengths of the correctors upstream of the buncher. The result is shown for a $100 \mathrm{pC}$ beam emitted $2.0 \mathrm{~mm}$ off-axis. Space charge is not included.

the buncher cavity, and of the accelerating cavities, as well as the solenoid magnetic field strengths.

During this optimization, space charge was turned off for simplicity. This was motivated by the consideration that the emittance growth in the off-axis beam is dominated by time-dependent rf effects. As a result, the optimized corrector settings obtained without space charge should be reasonably effective at removing these effects when space charge is included. This assumption will be verified in the next subsection, where simulation results with space charge are described.

The MOGA optimization process does not generate a single solution, but instead a set of solutions distributed along the so-called Pareto front, where each solution represents a different tradeoff between the objectives to be minimized [22]. In our case, the normalized horizontal and vertical rms emittances were chosen as the two objectives, and the correctors' strengths as the optimization knobs. The Pareto front generated by the MOGA optimization is shown in Fig. 7. The point indicated by the arrow corresponds to the solution that minimizes the geometric mean between the horizontal and vertical emittances, given by a value of $0.207 \mu \mathrm{m}$. By comparing this value with the corresponding values of $0.312 \mu \mathrm{m}$ for the off-axis uncorrected beam and $0.196 \mu \mathrm{m}$ for the beam emitted on-axis, we see that the optimization scheme can almost entirely remove the emittance growth due to off-axis emission when space charge is not included.

In the ASTRA simulations, the correctors were represented by short hard-edge dipoles with a fixed length of $10 \mathrm{~cm}$, and with field defined by the corresponding bending radius. 
TABLE IV. Bending radii for the correctors shown in Fig. 1, chosen to minimize the final beam emittance without space charge for a beam emitted $2 \mathrm{~mm}$ off-axis.

\begin{tabular}{lc}
\hline \hline Dipole corrector & Bending radius $(\mathrm{m})$ \\
\hline $\mathbb{A}$ in horizontal & 0.643 \\
$\mathbb{A}$ in vertical & 99.876 \\
$\mathbb{B}$ in horizontal & 14.889 \\
$\mathbb{B}$ in vertical & 9.998 \\
$\mathbb{C}$ in horizontal & -11.582 \\
$\mathbb{C}$ in vertical & 51.141 \\
$\mathbb{D}$ in horizontal & -0.996 \\
$\mathbb{D}$ in vertical & -1.242 \\
\hline \hline
\end{tabular}

Table IV contains a list of the correctors' bending radii associated with this optimized solution. Different signs of the bending radius represent different bending directions. Simulation in ASTRA of this optimized solution shows that the beam is not centered along the beam line components. Instead, the beam propagates off-axis through the buncher and the accelerating cavities, and remains off-axis up to the exit of the injector. This confirms, at least for the no-space charge case, the prediction that the emittance growth experienced by an off-axis beam in the gun can be compensated by properly displacing the beam in the downstream rf cavities.

Figures 8-9 show the results of the simulated optimization process for five different beam offsets in the gun (induced by different displacements of the laser on the photocathode). Figure 8 shows the beam offsets required in the buncher and in the first three accelerating cavities to properly compensate for the rf-induced emittance growth. A clear linear dependence of these offsets with respect to the initial laser displacement is visible, as expected by the theoretical model of (11). The figure also shows that

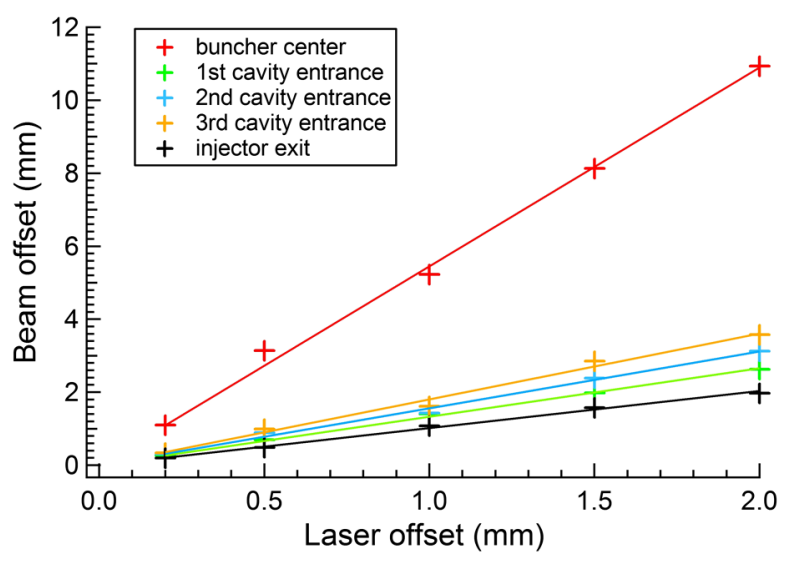

FIG. 8. The beam offset required in each cavity to compensate for the emittance growth due to off-axis beam emission is shown for different values of the initial laser offset (crosses). The values of the optimal beam offset at the injector exit are shown as black crosses. The colored lines denote linear fits to the data.

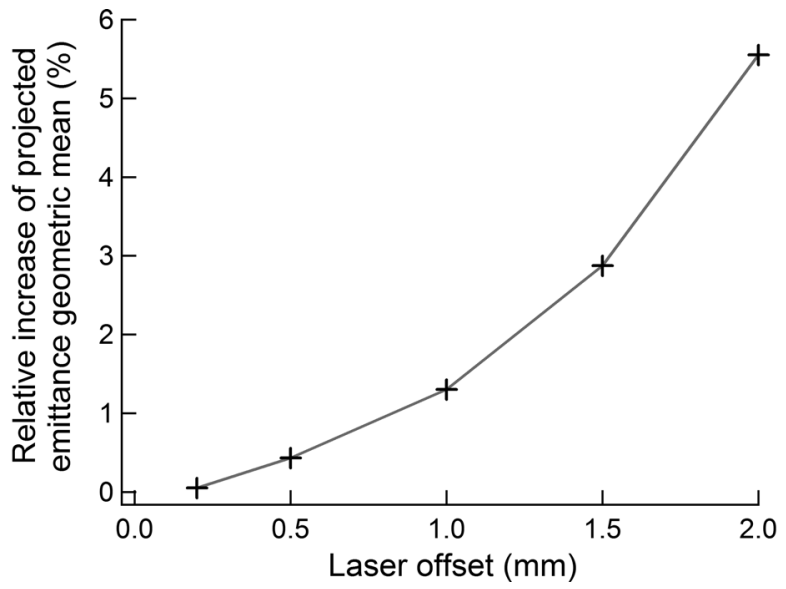

FIG. 9. Simulation results showing the residual emittance growth (after the compensation technique is applied) as a function of the initial laser offset at the cathode. Wakefield effects not included.

practical considerations, such as vacuum chamber stay-clear, could limit the use of very large offsets at the cathode because of the large orbit that could be required in some elements (the buncher in this particular example) to compensate for the emittance growth. For the purpose of this paper, the case of $2 \mathrm{~mm}$ offset at the cathode will be used. Such a value is compatible with the buncher and accelerating section apertures shown in Table I but, as it will be discussed later in the paper, it could be too large because of the effects induced by long range transverse wakefields on emittance at very high repetition rates.

Figure 9 shows the residual emittance growth after the compensation process has been applied for different laser offsets at the cathode. The results indicate that even for very large offsets at the cathode ( $2 \mathrm{~mm}$ case) the residual emittance growth can be contained by the compensation process to less than $6 \%$. This residual emittance growth is due to small additional linear and nonlinear coupling that cannot be removed by simple time-dependent rf focusing.

Additional simulations also show that the beam at the exit of the injector is sufficiently relativistic (energy $\sim 100 \mathrm{MeV}$ )

TABLE V. Final transverse emittances obtained using simulation with an axisymmetric $2 \mathrm{D}$ space charge solver and with a fully $3 \mathrm{D}$ space charge solver. The cases of on-axis emission and $2 \mathrm{~mm}$ off-axis emission with $250 \mathrm{~K}$ macroparticles are both listed. The off-axis beam is not corrected. (The dipole correctors are powered off).

\begin{tabular}{lccc}
\hline \hline Emission & $\begin{array}{c}\text { Emittance } \\
(\mu \mathrm{m})\end{array}$ & $\begin{array}{c}\text { 3D space } \\
\text { charge }\end{array}$ & $\begin{array}{c}\text { 2D }(\mathrm{R}-\mathrm{Z}) \text { space } \\
\text { charge }\end{array}$ \\
\hline \multirow{2}{*}{ On-axis } & $\varepsilon_{x}$ & 0.342 & 0.347 \\
& $\varepsilon_{y}$ & 0.342 & 0.348 \\
Off-axis & $\varepsilon_{x}$ & 0.410 & 1.533 \\
& $\varepsilon_{y}$ & 0.916 & 2.245 \\
\hline \hline
\end{tabular}




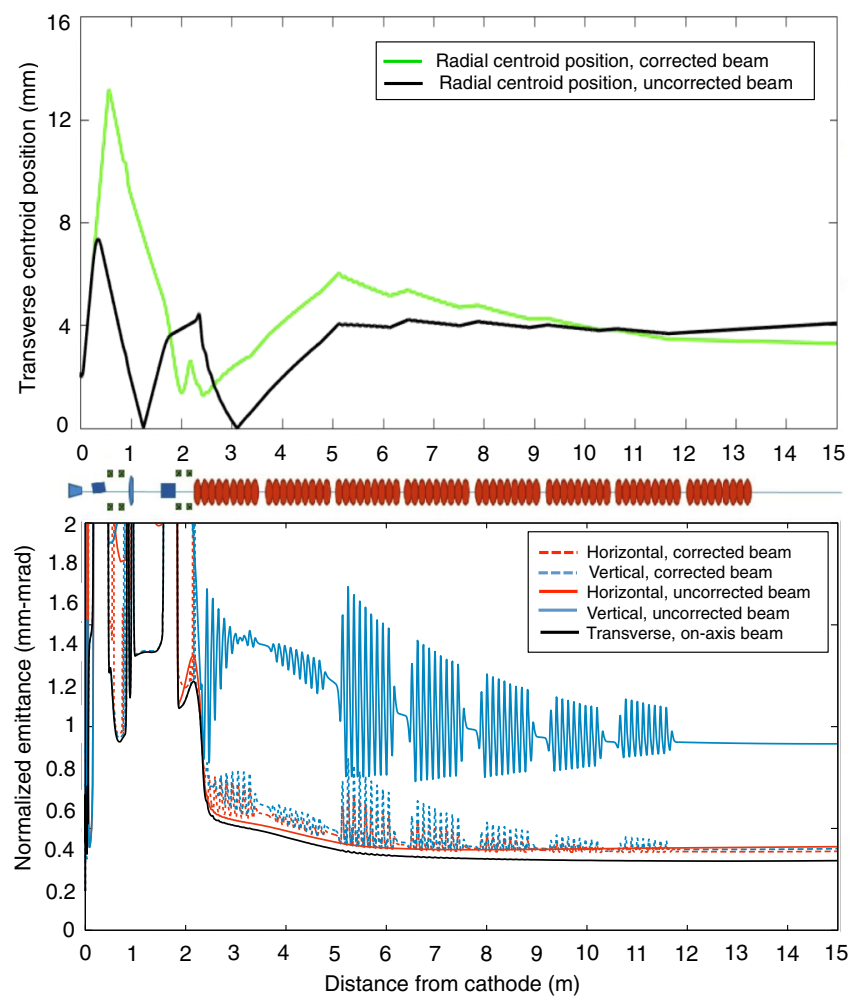

FIG. 10. Simulation of a beam emitted $2 \mathrm{~mm}$ off-axis at the cathode, with space charge included. Top: Beam centroid position along the beam line for the optimally corrected (green line) and noncorrected (black line) cases. Bottom: Normalized transverse emittance evolution along the beam line for the optimally corrected (dashed lines) and noncorrected (solid lines) cases. Red and blue curves are used to identify the horizontal and vertical planes, respectively.

to be realigned back along the downstream beam line axis without any further degradation of the emittance.

\section{Including space charge effects}

So far, the compensation procedure was simulated without space charge, using the code ASTRA combined with a MOGA optimization tool. ASTRA contains both 2D and 3D space charge algorithms. The 2D space charge algorithm is fast and efficient, but it does not represent an ideal match for our specific problem, where cylindrical symmetry is broken from the very beginning by the beam offset at the cathode. Using the 2D space charge algorithm in this situation can introduce artificial nonlinear contributions to the space charge field that will affect the accuracy of the final results. For simulations using 3D space charge, we chose to use the code IMPACT-T, due to its fast and very efficiently parallelized $3 \mathrm{D}$ solver.

To illustrate the importance of including 3D space charge, simulations of the photoinjector system were performed using IMPACT-T with 3D space charge included, and the results were compared against simulations performed using the 2D space charge algorithm in ASTRA. Parameters describing the initial beam distribution are provided in Table II. Table V shows the final emittances for a beam emitted on-axis and for a beam emitted $2 \mathrm{~mm}$ off-axis, with the dipole correctors powered off. The values of the on-axis beam emittance are in reasonable agreement, while there is a large difference between the values of the off-axis beam emittance, indicating that a $2 \mathrm{D}$ axisymmetric space-charge algorithm is insufficient for this case.

The next step was to set the correctors' strengths to the compensating settings given in Table IV for the case without space charge, keeping all other parameters the same, and tracking the off-axis beam using IMPACT-T with space charge included. The final beam emittance achieved using this optimized correction is $0.388 \mu \mathrm{m}$ in the horizontal and $0.400 \mu \mathrm{m}$ in the vertical, respectively. These numbers, when compared with the emittance of the on-axis beam $(0.342 \mu \mathrm{m})$ indicate that the compensation scheme was capable of reducing the emittance growth to $\sim 15 \%$. This value is acceptably small, considering the extreme offset of $2 \mathrm{~mm}$ used in the simulations. The effect of the compensation is clearly visible in Fig. 10. The simulations also show that as expected, the beam centroid propagates along the same trajectory as in the case with space charge off.

Table VI summarizes the vertical emittance decomposition results with space charge included. The values for the first two contributions, $\varepsilon_{\perp}$ and $\varepsilon_{R}$, are similar for all three cases shown. However, the misalignment contribution $\varepsilon_{\text {int }}$ for the corrected off-axis beam is much smaller than the corresponding contribution for the uncorrected off-axis beam. The remaining difference between the emittance of the on-axis beam and the emittance of the corrected

TABLE VI. Decomposition of the final vertical projected emittance for the off-axis beam, both with and without correction. The corresponding result for the on-axis beam is shown for comparison. Space charge is included in each simulation.

\begin{tabular}{lccc}
\hline \hline Emittance contribution $(\mu \mathrm{m})$ & On-axis beam & Off-axis beam without correction & Off-axis corrected beam \\
\hline Projected emittance $\varepsilon$ & 0.3995 & 0.9923 & 0.4657 \\
Mean slice $\varepsilon_{\perp}$ & 0.3332 & 0.3920 & 0.3443 \\
Mismatch $\varepsilon_{R}$ & 0.2178 & 0.2420 & 0.2262 \\
Linear correlation $\varepsilon_{\text {int }}$ & 0.0337 & 0.8761 & 0.2169 \\
Nonlinear correlation $\varepsilon_{\|}$ & 0.0011 & 0.0070 & 0.0116 \\
\hline \hline
\end{tabular}


off-axis beam is mostly due to residual misalignment of the slices. This suggests that space charge is affecting the transverse-longitudinal correlations in the beam and that an additional fine tuning of the correctors could be applied.

\section{WAKEFIELD EFFECTS}

The fields associated with an electron beam in an accelerator interact with the surrounding vacuum chamber, exciting wakefields that can interact back with the other particles in the bunch (short range wakefields), and in some cases with the following bunches (long range wakefields). Longitudinal (monopole) wakefields lead to beam energy loss, bunch longitudinal profile modifications, and increased energy spread along the bunch. An off-axis beam in a rf cavity can also excite transverse (dipole) wakefields. As a result, significant emittance growth or even beam break up may occur, reducing the brightness and introducing orbit errors. Because of this, wakefields can potentially affect the compensation mechanism described in the previous sections, and their effect needs to be carefully evaluated.

First, the effects of short-range longitudinal wakefields associated with the rf structures of the photoinjector beam line were evaluated. IMPACT-T simulations, including the wakefields for the buncher [23] and for the TESLA cavities [24], showed that the effects of such fields on the beam emitted off-axis are negligible.

Using a similar approach, IMPACT-T was also used to simulate the effects of short-range transverse wakefields in the TESLA cavities [24]. For a beam emitted $2 \mathrm{~mm}$ off-axis at the cathode, the emittance growth associated with the wakefields was less than $1 \%$, and the rms beam size along the injector was practically unaffected. The simulation results also showed that the beam centroid orbit and transverse-longitudinal correlations along the beam line were barely affected by the presence of the wakefields. For the buncher cavity case, since the geometry of its cells is similar to the TESLA cavity cells, and its length is much shorter than the TESLA cavities, the short range transverse wakefield effects could be safely assumed negligible.

The effects of long range transverse wakefields for the buncher [23] and the TESLA cavity $[25,26]$ were estimated analytically according to the procedure described in [27]. The results show that for repetition rates of up to $\sim 100 \mathrm{kHz}$, long range wakefield effects are negligible, but that for higher repetition rates the effects can become significant and must be taken into account. For example, for a $100 \mathrm{pC}$ beam with $2 \mathrm{~mm}$ offset at the cathode, the long range wakefields combined with the large beam orbit (required by the compensation technique inside the buncher and the accelerating sections) can generate at $1 \mathrm{MHz}$ repetition rate a significant emittance increase. In the worst case, assuming full coupling between the wakefields and the beam power spectrum, and the highest measured quality factors for the TESLA cavity wakefields, an emittance increase of $\sim 50 \%$ with respect to the on-axis beam case was estimated. Before these techniques are applied at high repetition rates, detailed simulation studies are suggested to investigate the effects of long range wakefields.

\section{CONCLUSIONS}

In certain circumstances, it is desirable to operate photoguns by stimulating electron emission from an area displaced from the cathode center. In the particular case of rf guns, the resulting orbit inside the gun cavity induces time-depending focusing of the beam that can create transverse-longitudinal correlations within the bunch, leading to an increase in the projected emittances. In this paper, we have described the physics behind this phenomenon, and we defined a scheme for compensating the emittance increase by forcing the beam to lie on a properly-chosen off-axis trajectory in the downstream rf structures. Such a configuration allows the additional time-dependent focusing to remove the bunch correlations, and reduces the projected emittance values to those obtained for a beam emitted on-axis. The compensation scheme was benchmarked by a series of simulations, and the results were presented. The effects of wakefields on the compensation process were also evaluated showing that at $\mathrm{MHz}$-class repetition rates, long range transverse wakefields in the injector rf cavities can limit the applicability of the compensation method for large beam offsets at the cathode.

\section{ACKNOWLEDGMENTS}

This work was supported by the Director of the Office of Science of the US Department of Energy under Contract No. DEAC02-05CH11231.

[1] C. Pellegrini, in Proceedings of the 2nd International Particle Accelerator Conference, San Sebastián, Spain (EPS-AG, Spain, 2011), p. 3798.

[2] Y. Shen, X. Yang, G. L. Carr, Y. Hidaka, J. B. Murphy, and X. Wang, Tunable Few-Cycle and Multicycle Coherent Terahertz Radiation from Relativistic Electrons, Phys. Rev. Lett. 107, 204801 (2011).

[3] P. Musumeci, J. T. Moody, C. M. Scoby, M. S. Gutierrez, and M. Westfall, Laser-induced melting of a single crystal gold sample by time-resolved ultrafast relativistic electron diffraction, Appl. Phys. Lett. 97, 063502 (2010).

[4] X. F. D. Stragier, O. J. Luiten, S. B. van der Geer, M. J. van der Wiel, and G. J. H. Brussaard, Experimental validation of a radio frequency photogun as external electron injector for a laser wakefield accelerator, J. Appl. Phys. 110, 024910 (2011).

[5] J. Lewellen, Ion tracking in photocathode rf guns, Phys. Rev. ST Accel. Beams 5, 020101 (2002).

[6] J. Qiang, Particle-in-cell/Monte Carlo simulation of ion back bombardment in a high average current RF 
photo-gun, Nucl. Instrum. Methods Phys. Res., Sect. A 614, 1 (2010).

[7] L. Cultrera et al., Photocathode behavior during high current running in the Cornell energy recovery linac photoinjector, Phys. Rev. ST Accel. Beams 14, 120101 (2011).

[8] B. Dunham et al., Record high-average current from a high-brightness photoinjector, Appl. Phys. Lett. 102, 034105 (2013).

[9] F. Sannibale et al., Advanced photoinjector experiment photogun commissioning results, Phys. Rev. ST Accel. Beams 15, 103501 (2012).

[10] K.-J. Kim, Rf and space-charge effects in laser-driven rf electron guns, Nucl. Instrum. Methods Phys. Res., Sect. A 275, 201 (1989).

[11] T.P. Wangler, RF Linear Accelerators, 2nd ed. (Wiley-VCH, Weinheim, 2008), p. 168.

[12] D. T. Abell, Numerical computation of high-order transfer maps for rf cavities, Phys. Rev. ST Accel. Beams 9, 052001 (2006).

[13] L. Serafini, Beam dynamics in rf guns and emittance correction techniques, Nucl. Instrum. Methods Phys. Res., Sect. A 340, 40 (1994).

[14] I. V. Bazarov, A. Kim, M. N. Lakshmanan, and J. M. Maxson, Comparison of dc and superconducting rf photoemission guns for high brightness high average current beam production, Phys. Rev. ST Accel. Beams 14, 072001 (2011).

[15] B. Aune et al., Superconducting TESLA cavities, Phys. Rev. ST Accel. Beams 3, 092001 (2000).

[16] C.F. Papadopoulos et al., in Proceedings of the 5th International Particle Accelerator Conference, Dresden, Germany (JACoW, Geneva, Switzerland, 2014), p. 1974.
[17] K. Deb, A fast and elitist multiobjective genetic algorithm: NSGA-II, IEEE Trans. Evol. Comput. 6, 182 (2002).

[18] K. Flottman, ASTRA: A Space Charge Tracking Algorithm, http://www.desy.de/ mpyflo/Astra_manual/ Astra-Manual_V3.1.pdf.

[19] J. Qiang, S. Lidia, R. D. Ryne, and C. Limborg-Deprey, Three-dimensional quasistatic model for high brightness beam dynamics simulation, Phys. Rev. ST Accel. Beams 9 , 044204 (2006).

[20] C. Mitchell, A general slice moment decomposition of RMS beam emittance, arXiv:1509.04765; Lawrence Berkeley National Laboratory Report No. LBNL-6996E, 2015.

[21] C. Mitchell, J. Qiang, and P. Emma, Longitudinal pulse shaping for the suppression of coherent synchrotron radiation-induced emittance growth, Phys. Rev. ST Accel. Beams 16, 060703 (2013).

[22] K. Deb, Multi-Objective Optimization Using Evolutionary Algorithms (John Wiley \& Sons, Inc., New York, 2001).

[23] H. Qian et al., in Proceedings of the 5th International Particle Accelerator Conference, Dresden, Germany (JACoW, Geneva, Switzerland, 2014), p. 3924.

[24] T. Weiland and I. Zagorodnov, TESLA Report No. 200319, 2003.

[25] N. Baboi et al., in Proceedings of the European Particle Accelerator Conference, Vienna, 2000 (EPS, Geneva, 2000), p. 1107.

[26] R. Wanzenberg, TESLA Report No. 2001-33, 2001.

[27] H. Padamsee, J. Knobloch, and T. Hays, RF Superconductivity for Accelerators (Wiley, New York, 1998), p. 334. 